OPEN ACCESS

Edited by:

Lucia Tabares,

University of Seville, Spain

Reviewed by:

Sandy Bajjalieh,

University of Washington School of

Medicine, USA

Peter Juo,

Tufts University, USA

*Correspondence:

Anna Fassio

afassio@unige.it;

Fabio Benfenati

fabio.benfenati@iit.it

Received: 12 February 2016 Accepted: 18 April 2016

Published: 12 May 2016

Citation:

Fassio A, Fadda M and Benfenati F (2016) Molecular Machines Determining the Fate of Endocytosed Synaptic Vesicles in Nerve Terminals. Front. Synaptic Neurosci. 8:10. doi: 10.3389/fnsyn.2016.00010

\section{Molecular Machines Determining the Fate of Endocytosed Synaptic Vesicles in Nerve Terminals}

\author{
Anna Fassio ${ }^{1,2 *}$, Manuela Fadda ${ }^{1}$ and Fabio Benfenati ${ }^{1,2 *}$ \\ ${ }^{1}$ Department of Experimental Medicine, University of Genoa, Genoa, Italy, ${ }^{2}$ Center of Synaptic Neuroscience and \\ Technology, Istituto Italiano di Tecnologia, Genova, Italy
}

The cycle of a synaptic vesicle (SV) within the nerve terminal is a step-by-step journey with the final goal of ensuring the proper synaptic strength under changing environmental conditions. The SV cycle is a precisely regulated membrane traffic event in cells and, because of this, a plethora of membrane-bound and cytosolic proteins are devoted to assist SVs in each step of the journey. The cycling fate of endocytosed SVs determines both the availability for subsequent rounds of release and the lifetime of SVs in the terminal and is therefore crucial for synaptic function and plasticity. Molecular players that determine the destiny of SVs in nerve terminals after a round of exo-endocytosis are largely unknown. Here we review the functional role in SV fate of phosphorylation/dephosphorylation of SV proteins and of small GTPases acting on membrane trafficking at the synapse, as they are emerging as key molecules in determining the recycling route of SVs within the nerve terminal. In particular, we focus on: (i) the cyclin-dependent kinase-5 (cdk5) and calcineurin (CN) control of the recycling pool of SVs; (ii) the role of small GTPases of the Rab and ADP-ribosylation factor (Arf) families in defining the route followed by SV in their nerve terminal cycle. These regulatory proteins together with their synaptic regulators and effectors, are molecular nanomachines mediating homeostatic responses in synaptic plasticity and potential targets of drugs modulating the efficiency of synaptic transmission.

Keywords: presynapse, synaptic vesicle, cdk5, calcineurin, recycling, small GTPases, Rab, Arf

\section{INTRODUCTION}

The synaptic vesicle (SV) cycle is the most highly regulated membrane traffic event in cells and the proteins involved, as well as the arrays of protein-protein interactions that guarantee the fidelity of the process, are getting increasingly clear.

Kinases and phosphatases, as well as small GTPases and their effectors, are emerging as molecular machines acting at the synapse to regulate synaptic function and plasticity by rapidly modulating several synaptic targets and adapting the synapse to the needs of the network.

The high tunability of synaptic strength is obtained presynaptically from changes in quantal size, SV availability for release and number of active synapses, and all these parameters are directly or indirectly controlled by the cycling fate of SVs.

Here we provide an update on two molecular machines recently reported to act on the fate of recycling SVs in the nerve terminal: the cyclin-dependent kinase $5(\mathrm{cdk} 5) /$ calcineurin $(\mathrm{CN})$ 
system with its multiple synaptic substrates and the small GTPase Rab and ADP ribosylation factor (Arf) systems with their relative regulators and effectors.

\section{THE cdk5/CN SYSTEM}

Kinases and phosphatases post-translationally regulate the function of a plethora of proteins and play a major role in regulating cellular functions. Phosphoproteins are highly expressed at the synaptic terminal together with dedicated kinases and phosphatases, and their activity regulates many aspects of SV cycling. The role of phosphoproteins in SV cycling has been extensively reviewed elsewhere (Valtorta and Benfenati, 1994). We here focus on one of the presynaptically expressed kinases, cdk5, and the cognate phosphatase, CN, as they act as key molecules in regulating the strength of neurotransmission by acting on SVs availability for release and endocytic recovery of SV at the plasma membrane after exocytosis.

The first evidence for a central role of the $\mathrm{cdk} 5 / \mathrm{CN}$ system at the presynapse derives from the observation that several proteins involved in various steps of the SV cycle are specific substrates for $c d k 5 / \mathrm{CN}$, namely munc18-1, septin5, Pictaire, synapsin, amphiphysin, dynamin and synaptojanin (reviewed in $\mathrm{Su}$ and Tsai, 2011). From a functional point of view, it is possible to distinguish between effects on exocytosis and endocytosis, keeping in mind that the two processes are intimately connected and both regulated by calcium waves in the terminal ( $\mathrm{Wu}$ et al., 2014; Leitz and Kavalali, 2015). SV exocytosis is regulated by cdk 5 phosphorylation of Munc18 that allows syntaxin 1 to participate in the SNARE complex and SV fusion to occur (Shuang et al., 1998; Fletcher et al., 1999). A similar effect has been described for the phosphorylation of the cytoskeletal protein septin 5 by $\mathrm{cdk} 5$, which decreases binding of septin 5 to the SNARE protein syntaxin1 and regulates neurotransmitter release (Taniguchi et al., 2007; Amin et al., 2008). The cdk5 substrate Pictaire has also been proposed to regulate exocytosis by phosphorylating N-Ethylmaleimide-Sensitive Factor (NSF) and regulating the ability of NSF to oligomerize (Liu et al., 2006). Additional roles for cdk5/CN in the process of exocytosis came from the observation, made both in vitro and in vivo, that N-type calcium channels (Cav2.2) are substrates for the two enzymes (Su et al., 2012; Kim and Ryan, 2013). Although the impact of phosphorylation on the properties of the Cav2.2 channel is still controversial, it has been nicely demonstrated that the $c \mathrm{dk} 5 / \mathrm{CN}$ balance regulates the final steps of exocytosis by potently controlling the action potential-driven calcium influx and therefore the probability of release (Kim and Ryan, 2013).

On the endocytosis side, an array of $\mathrm{CN}$ substrates involved in the SV retrieval process are known among the main molecular actors of SV cycling (Marks and McMahon, 1998). These proteins have been collectively named dephosphins, as their dephosphorylation is induced by calcium increase during stimulation (Cousin and Robinson, 2001). Both their dephosphorylation and subsequent phosphorylation by cdk 5 is required for SV retrieval in central nerve terminals
(Clayton et al., 2007). Different mechanisms of SV endocytosis operate at central synapses, namely fast endocytosis, clathrindependent endocytosis and bulk endocytosis (Kononenko and Haucke, 2015). However, how the various modes of endocytosis are interconnected and differently regulated is still a matter of investigation. The cdk5/CN activity on dephosphins has been reported to selectively regulate slow, activity-dependent bulk endocytosis, with no effect on fast endocytosis. The slow form of endocytosis predominantly occurs during sustained activity, requires traffic of SVs via the endosomal compartment and repopulates the recycling pool, which is only released during intense stimulation after the complete depletion of the readily releasable pool (Evans and Cousin, 2007; Cheung and Cousin, 2013). Interestingly, cdk5/CN activities concomitantly control which fraction of SVs partitions into the recycling or the releasereluctant resting pool (Kim and Ryan, 2010; Marra et al., 2012) and the SV protein Synapsin I has been identified as the main $c d k 5 / C N$ substrate in mediating this effect (Verstegen et al., 2014). In particular, cdk5-phopshorylated Synapsin I sequesters recycling SVs in the release-reluctant resting pool by clustering SVs and increasing their association with actin filaments. The dual effect on bulk endocytosis and SV pool partitioning suggests that the system is involved in determining the fate of endocytosed SVs; in particular, it seems that during sustained activity bulk endocytosis proceeds via sequential $\mathrm{CN} / \mathrm{cdk} 5$ activation, resulting in endosomal recycling of SVs and in the capture of the newly formed SVs into the release-reluctant pool (Figure 1). Moreover, the balance of $\mathrm{cdk} 5 / \mathrm{CN}$ activities broadly varies between synapses, setting both the tone of N-type calcium channels and the ratio of recycling vs. reluctant SVs and resulting in synapse heterogeneity from silent to strongly active synapses (Kim and Ryan, 2010, 2013; Verstegen et al., 2014).

As a result of the multiple targets at the presynapse, the $\mathrm{cdk} 5 / \mathrm{CN}$ nanomachine represents a master regulator of synaptic homeostasis and participates in the scaling of synaptic strength to compensate for the effects of sustained hypo- or hyperactivity (Seeburg et al., 2008; Kim and Ryan, 2010; Mitra et al., 2011; Peng et al., 2013). Chronic silencing of firing strongly downregulates nerve terminal cdk 5 with the result of recruiting release reluctant SVs to the recycling pool. This process, which appears to be mediated by changes in the phosphorylation state of Synapsin I, can activate previously silent synapses and change the release potential of already active synapses (Kim and Ryan, 2010; Verstegen et al., 2014). Alterations of cdk5 activity are associated with a broad range of neurological disorders (see for reviews Cheung and Ip, 2012; McLinden et al., 2012) and in particular the cdk5-mediated homeostatic synaptic response has been recently involved in early Alzheimer's like synaptic pathology (Sheng et al., 2015).

\section{Rab AND Arf SYSTEMS}

Rab and Arf family proteins are master regulators of membrane trafficking and are involved in all steps of vesicular transport. As all GTPases, these proteins function as molecular switches by cycling between active guanosine triphosphate (GTP)-bound 


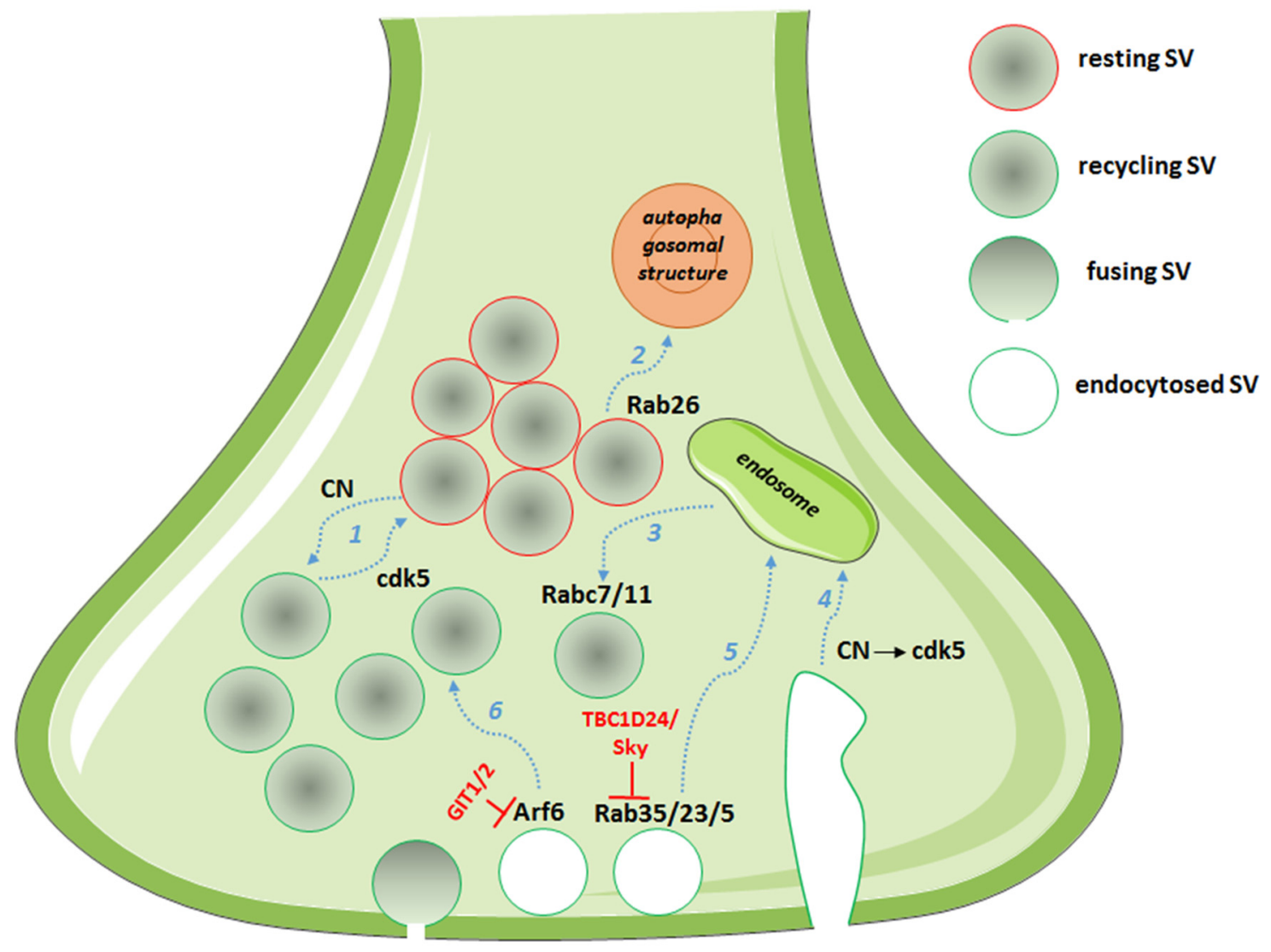

FIGURE 1 | Picture showing the multiple roles of cyclin-dependent kinase 5 (cdk5)/calcineurin (CN) and Rab/ADP-ribosylation factor (Arf) system in defining the route for endocytosed SVs. Dashed arrows in light blue represent synaptic vesicle (SV) routes: (1) partitioning into pools; (2) degradation; (3) post-endosomal trafficking; (4) endosomal sorting after bulk endocytosis; (5) endosomal trafficking; and (6) direct recycling. In red, the GTPase activating proteins (GAPs) with the described functions in controlling the SV fate.

and inactive guanosine diphosphate (GDP)-bound states. Their cycling is regulated by two families of regulatory proteins, namely guanine nucleotide exchange factors (GEFs) and GTPase activating proteins (GAPs). GEFs function as activators by facilitating the conversion from a GDP-bound form to a GTP bound form, whereas GAPs function as repressors by enhancing GTP hydrolysis. The vast number of Rab and Arf proteins and the multiple GAPs and GEFs for each isoform make the small GTPase family an ideal spatial and temporal regulator of virtually every aspect of membrane trafficking (Stenmark, 2009). As SV cycling is the prototype of an intensely regulated membrane traffic event, Rab and Arf proteins, together with their regulators and effectors, are emerging as molecular nanomachines regulating specific steps of the cycle.

More than 30 distinct Rabs were indeed identified by proteomic analysis in highly purified SV fractions (Takamori et al., 2006) and the three more abundant Rabs (Rab3, 7 and 5) were quantified in isolated terminals and found to represent $0.776,0.135$ and $0.025 \%$ of total synaptic protein, a percentage comparable with other fundamental protein for presynaptic physiology such as, for example, the calcium sensor synaptotagmin1 (Wilhelm et al., 2014). The role of the Rabs involved in exocytosis, such as the secretory Rabs Rab3a/3b/3c and Rab27b, and the role of some of the Rabs involved in SV recycling, such as Rab4, 5, 10,11b and 14, has been reviewed and recently commented elsewhere (Sudhof, 2004; Pavlos et al., 2010; Pavlos and Jahn, 2011; Giorgini and Steinert, 2013; Rizzoli, 2014). Here we focus on additional small GTPases recently described to participate in SV cycling, particularly in the steps defining fate of SVs after recovery.

\section{Rab35}

A role for Rab35 in SV cycling originated from the identification of TBC1D24, an epilepsy gene involved in neuronal development and protein partner of the small GTPase Arf6 (Corbett et al., 2010; Falace et al., 2010, 2014). Studying the Drosophila mutants for TBC1D24/Skywalker, Uytterhoeven et al. (2011) revealed a strong presynaptic phenotype with a larger readily releasable pool of SVs and a dramatic increase in basal neurotransmitter release at neuromuscular junction (NMJ) synapses. They reported TBC1D24 to act as a GAP for Rab35 and proposed that active GTP-bound Rab35 favors endosomal sorting of SV proteins and replacement of dysfunctional SV components. Rab5 and 
TABLE 1 | Small GTPases playing a role at the presynaptic terminal.

\begin{tabular}{|c|c|c|c|}
\hline Small GTPases & Presynaptic role & Presynaptic GAP and GEF & Reference \\
\hline Rab3a,b,c & Docking/priming & RAb3GAP, Rab3GEP... & $\begin{array}{l}\text { Geppert et al. (1994, 1997), Yamaguchi et al. (2002), Giovedì et al. } \\
\text { (2004a,b), Schlüter et al. (2004), Sakane et al. (2006), Pavlos et al. (2010), } \\
\text { and Pavlos and Jahn (2011) }\end{array}$ \\
\hline Rab4 & SV recycling/sorting & To be determined & Pavlos and Jahn (2011) \\
\hline Rab5a,b & SV recycling/sorting & To be determined & $\begin{array}{l}\text { de Hoop et al. (1994), Fischer von Mollard et al. (1994), Shimizu et al. } \\
\text { (2003), Wucherpfennig et al. (2003), Star et al. (2005), Hoopmann et al. } \\
\text { (2010), Pavlos and Jahn (2011), and Uytterhoeven et al. (2011) }\end{array}$ \\
\hline Rab7 & SV recycling/sorting & To be determined & Pavlos and Jahn (2011) and Uytterhoeven et al. (2011) \\
\hline Rab10 & SV recycling/sorting & To be determined & Pavlos and Jahn (2011) \\
\hline Rab11b & SV recycling/sorting & To be determined & $\begin{array}{l}\text { Pavlos and Jahn (2011), Steinert et al. (2012), and Giorgini and Steinert } \\
\text { (2013) }\end{array}$ \\
\hline Rab14 & SV recycling/sorting & To be determined & Pavlos and Jahn (2011) \\
\hline Rab23 & SV recycling/sorting & To be determined & Uytterhoeven et al. (2011) \\
\hline Rab26 & SV degradation & To be determined & Binotti et al. (2015) \\
\hline Rab27b & Docking/priming & To be determined & Pavlos et al. (2010) and Pavlos and Jahn (2011) \\
\hline Rab35 & SV recycling/sorting & TBC1D24, Connecden1... & Allaire et al. (2006) and Uytterhoeven et al. (2011) \\
\hline Arf1 & SV budding & Arf1GAP... & Faúndez et al. (1997) \\
\hline Arf6 & SV recycling/sorting & GIT, Centaurin... & $\begin{array}{l}\text { Ashery et al. (1999), Krauss et al. (2003), Homma et al. (2014), Podufall } \\
\text { et al. (2014), Montesinos et al. (2015), and Tagliatti et al. (2016) }\end{array}$ \\
\hline Arl8 & SV component transport & To be determined & Klassen et al. (2010) and Wu et al. (2013) \\
\hline
\end{tabular}

Rab23 were also described to exert similar effects on endosomal trafficking of SVs, although TBC1D24 seems to act as a selective GAP for Rab35. This is the first description of a synaptic machinery dedicated to the control of the quality of proteins in SV cycling (Figure 1, Uytterhoeven et al., 2011). Moreover, using a functional screen to assess the impact of a battery of constitutively active Rabs on SV cycling, the same authors identified Rab7 and Rab11 as putative regulators of postendosomal trafficking of SVs (Figure 1, Uytterhoeven et al., 2011). Whether a similar mechanism also operates at mammalian central synapses remains to be investigated.

\section{Rab26}

By investigating the molecular mechanisms leading to synapse elimination, Binotti et al. (2015) recently described a role for the small GTPase Rab26 in directing SVs into preautophagosomal structures, thus proposing a novel pathway for the degradation of SVs. The small GTPase was found in a subset of presynaptic terminals associated with marked clustering of SVs and is believed to promote SV clustering via a still unknown mechanism. Rab26, in its active GTP-bound state, was also reported to recruit Atg16L1 as an effector, representing a link between SV cycling and autophagy (Figure 1, Binotti et al., 2015).

\section{Arf}

The Arf proteins are a family of six small, ubiquitously expressed GTP-binding proteins (Donaldson and Jackson, 2011) that can be divided into three classes, based on sequence identity. Class I
Arf proteins (Arf1, Arf2 and Arf3) regulate the assembly of various types of "coat" complexes onto budding vesicles along the secretory pathway and activate lipid-modifying enzymes (Bonifacino, 2004); Class II Arf proteins (Arf4 and Arf5), whose function is still unclear; and Arf6, which is the sole member of class III Arf proteins known to regulate endosomal membrane traffic and structural organization at the cell surface (D'Souza-Schorey and Chavrier, 2006). Other proteins that structurally resemble Arf proteins are the Arf-like (Arl) proteins, the Ras-related protein-1 (SAR1p) and the Arf-related protein ARFRP1.

Although Arf proteins are less abundant in purified SVs or isolated nerve terminals as compared to other small GTPases, the Arf analogs Arl10b and Arl10c and the Arf-interacting protein arfaptin 2 have been identified as components of purified SVs (Takamori et al., 2006). Some of the Arf proteins have been described to play a role in the definition of SV fate at the synapse.

The class III Arf protein Arf6, involved in constitutive trafficking between the plasma membrane and early endosomes and actin dynamics, has been reported to increase basal synaptic transmission at the Xenopus NMJ (Ashery et al., 1999), to regulate the assembly of the clathrin-coat complex during SV endocytosis (Krauss et al., 2003) and to play a role in the SV recycling pathway (Tagliatti et al., 2016). Knockdown of Arf6 in rat hippocampal neurons results in a strong presynaptic phenotype, with decreased SV density, accumulation of endosomal structures in the terminal and increased functional releasable SVs docked to the plasma membrane. Arf6 appears to act as a molecular determinant in the formation of the 
readily releasable pool of SVs and in the sorting of endocytosed SVs to direct recycling, rather than through the endosomal compartment (Tagliatti et al., 2016, Figure 1). Interestingly, the observed Arf6-knockdown phenotype is reminiscent of the synaptic phenotype for the constitutively active Rab35 at the Drosophila NMJ (Uytterhoeven et al., 2011), suggestive of a functional interplay of the two small GTPases at the presynaptic compartment, as reported in different cellular systems (Allaire et al., 2013; Miyamoto et al., 2014). Although no precise synaptic role for Arf1 has been proposed, the protein in its active GTP-bound form has been described to regulate vesicle budding in PC12 cells (Faúndez et al., 1997), suggesting that also the prototype class I Arf may act in SV cycling pathway.

In support for a presynaptic role for Arf proteins, the Arf-like small G protein, Arl-8, has been identified as a critical regulator of presynaptic patterning and axonal transport in C. elegans (Klassen et al., 2010) and active GTP-bound Arl-8 were reported to act as an effector of the anterograde motor UNC-104/KIF1A (Wu et al., 2013). The Arl-8 GTP/GDP cycle is therefore proposed as a switch to control the association/dissociation of SV precursors from microtubule motor proteins, thus ensuring the proper delivery of novel presynaptic components at nerve terminals (Wu et al., 2013).

\section{GAPs and GEFs}

Considering the prominent roles of Rab and Arf proteins at the synaptic terminal, it is noteworthy the lack of data on synaptic GAPs and GEFs that dynamically regulate their activity and potentially represent targets to finely modulate neurotransmission in both homeostatic and hebbian plasticity (Table 1). In addition to the above mentioned role for the Rab35GAP TBC1D24 (Uytterhoeven et al., 2011), the specific GAP for Arf6, G-protein coupled receptor kinase 2 interacting protein (GIT) has been involved in the organization of the cytomatrix of the active zone (Kim et al., 2003) and recently reported to play multiple roles at the presynapse (Podufall et al., 2014; Montesinos et al., 2015). Podufall et al. (2014) analyzed the localization of GIT at hippocampal glutamatergic synapses and at the Drosophila NMJ by employing SD-dSTORM high-resolution microscopy and revealed that the protein localizes at the periphery of the active zone. Indeed, GIT interacts with the endocytic adaptor stoninB and regulates the localization and function of stoninB at the presynaptic site. A Drosophila GIT mutant showed accumulation of endosomal structures and vacuoles and marked defects in post-stimulus SV endocytosis and/or re-acidification (Podufall et al., 2014). Although confirming a clear presynaptic function for the GAP of Arf6, studies in mammalian synapses revealed different roles for GIT1 and the other mammalian isoform GIT2. GIT1 and GIT1/GIT2 knocked out calyx of Held synapses showed a markedly increased initial release probability that was not associated with changes in the size of the readily releasable pool or in voltage-dependent calcium channel activity. Although some discrepancies in the reported results exist, and the precise mechanisms of the synaptic actions of GIT are still to be clarified (Montesinos et al., 2015), the small GTPase Arf6 and the two Arf6 regulators, TBC1D24 and GIT, are emerging as synaptic nanomachines operating at the presynaptic site to define both release probability and SV recycling pathways.

The ArfGAP1, acting as a GAP for Arf1, has been found to interact and regulate the activity of Leucine-rich repeat kinase 2 (LRRK2, Xiong et al., 2012), whose gene is mutated in Parkinson's disease patients, and known to play multiple roles in the presynaptic compartment (see for a review Belluzzi et al., 2012). LRRK2 kinase activity was also recently described to function as a Rab5b GAP, negatively regulating Rab5b signaling (Yun et al., 2015). In addition to LRRK2, huntingtin protein, dysfunctional in Huntigton's disease, has been proposed to function as a Rab regulator. Indeed, huntingtin was found in a complex acting as a GEF for Rab11 (Li et al., 2008), and Rab11 overexpression rescued the synaptic dysfunction associated with both huntingtin and synuclein mutations in Drosophila (Steinert et al., 2012; Breda et al., 2015).

\section{CONCLUDING REMARKS}

The cdk5/CN system is a master regulator of synaptic strength and synaptic functional heterogeneity by modulating calcium signaling and SV distribution in the nerve terminal. The system is highly regulated by homeostatic plasticity and represents a target for research on the molecular markers of synaptic dysfunctions and on the design of novel drugs (Shah and Lahiri, 2014; Sheng et al., 2015).

For the Rab/Arf system, we expect that additional GAP and GEF functions at the synapse will be clarified in the near future, together with the role of specific GTPase effectors in synaptic function. The synaptic targeting and/or the activity modulation of GAP and GEF will emerge as main factors in synaptic plasticity processes and in the pathogenesis of synaptic dysfunctions. Interestingly, several mutations in genes encoding proteins belonging to the Rab and Arf small GTPases families, or proteins regulating their GTP-binding cycle, have been recently described as causative for inherited neurological diseases (Falace et al., 2010; Shoubridge et al., 2010; Rauch et al., 2012; Seixas et al., 2013; D’Adamo et al., 2014; Fine et al., 2015; Kalscheuer et al., 2016), making the exploitation of their function at the synapse a prerequisite for the design of effective therapeutic strategies.

\section{AUTHOR CONTRIBUTIONS}

AF drafted the article and prepared the figure. MF assisted in drafting the article. FB drafted and revised the article.

\section{ACKNOWLEDGMENTS}

The authors apologize for all scientists whose important contributions were not cited due to space limitations. The work has been supported by Università degli Studi di Genova to AF, EU FP7 Integrating Project "Desire" (Grant no. 602531) to FB and $\mathrm{AF}$ is also acknowledged. 


\section{REFERENCES}

Allaire, P. D., Ritter, B., Thomas, S., Burman, J. L., Denisov, A. Y., LegendreGuillemin, V., et al. (2006). Connecdenn, a novel DENN domain-containing protein of neuronal clathrin-coated vesicles functioning in synaptic vesicle endocytosis. J. Neurosci. 26, 13202-13212. doi: 10.1523/jneurosci.4608-06. 2006

Allaire, P. D., Seyed Sadr, M., Chaineau, M., Seyed Sadr, E., Konefal, S., Fotouhi, M., et al. (2013). Interplay between Rab35 and Arf6 controls cargo recycling to coordinate cell adhesion and migration. J. Cell Sci. 126, 722-731. doi: 10. 1242/jcs. 112375

Amin, N. D., Zheng, Y. L., Kesavapany, S., Kanungo, J., Guszczynski, T., Sihag, R. K., et al. (2008). Cyclin-dependent kinase 5 phosphorylation of human septin SEPT5 (hCDCrel-1) modulates exocytosis. J. Neurosci. 28, 3631-3643. doi: 10. 1523/JNEUROSCI.0453-08.2008

Ashery, U., Koch, H., Scheuss, V., Brose, N., and Rettig, J. (1999). A presynaptic role for the ADP ribosylation factor (ARF)-specific GDP/GTP exchange factor msec7-1. Proc. Natl. Acad. Sci. U S A 96, 1094-1099. doi: 10.1073/pnas.96.3. 1094

Belluzzi, E., Greggio, E., and Piccoli, G. (2012). Presynaptic dysfunction in Parkinson's disease: a focus on LRRK2. Biochem. Soc. Trans. 40, 1111-1116. doi: 10.1042/bst20120124

Binotti, B., Pavlos, N. J., Riedel, D., Wenzel, D., Vorbrüggen, G., Schalk, A. M., et al. (2015). The GTPase Rab26 links synaptic vesicles to the autophagy pathway. Elife 4:e05597. doi: 10.7554/eLife.05597

Bonifacino, J. S. (2004). The GGA proteins: adaptors on the move. Nat. Rev. Mol. Cell Biol. 5, 23-32. doi: 10.1038/nrm1279

Breda, C., Nugent, M. L., Estranero, J. G., Kyriacou, C. P., Outeiro, T. F., Steinert, J. R., et al. (2015). Rab11 modulates $\alpha$-synuclein-mediated defects in synaptic transmission and behaviour. Hum. Mol. Genet. 24, 1077-1091. doi: 10. 1093/hmg/ddu521

Cheung, G., and Cousin, M. A. (2013). Synaptic vesicle generation from activitydependent bulk endosomes requires calcium and calcineurin. J. Neurosci. 33, 3370-3379. doi: 10.1523/jneurosci.4697-12.2013

Cheung, Z. H., and Ip, N. Y. (2012). Cdk5: a multifaceted kinase in neurodegenerative diseases. Trends Cell Biol. 22, 169-175. doi: 10.1016/j.tcb. 2011.11 .003

Clayton, E. L., Evans, G. J., and Cousin, M. A. (2007). Activity-dependent control of bulk endocytosis by protein dephosphorylation in central nerve terminals. J. Physiol. 585, 687-691. doi: 10.1113/jphysiol.2007.137539

Corbett, M. A., Bahlo, M., Jolly, L., Afawi, Z., Gardner, A. E., Oliver, K. L., et al. (2010). A focal epilepsy and intellectual disability syndrome is due to a mutation in TBC1D24. Am. J. Hum. Genet. 87, 371-375. doi: 10.1016/j.ajhg. 2010.08.001

Cousin, M. A., and Robinson, P. J. (2001). The dephosphins: dephosphorylation by calcineurin triggers synaptic vesicle endocytosis. Trends Neurosci. 24, 659-665. doi: 10.1016/s0166-2236(00)01930-5

D’Adamo, P., Masetti, M., Bianchi, V., Morè, L., Mignogna, M. L., Giannandrea, M., et al. (2014). RAB GTPases and RAB-interacting proteins and their role in the control of cognitive functions. Neurosci. Biobehav. Rev. 46, 302-314. doi: 10. 1016/j.neubiorev.2013.12.009

de Hoop, M. J., Huber, L. A., Stenmark, H., Williamson, E., Zerial, M., Parton, R. G., et al. (1994). The involvement of the small GTP-binding protein Rab5a in neuronal endocytosis. Neuron 13, 11-22. doi: 10.1016/0896-6273(94) 90456-1

Donaldson, J. G., and Jackson, C. L. (2011). ARF family G proteins and their regulators: roles in membrane transport, development and disease. Nat. Rev. Mol. Cell Biol. 12, 362-375. doi: 10.1038/nrm3117

D'Souza-Schorey, C., and Chavrier, P. (2006). ARF proteins: roles in membrane traffic and beyond. Nat. Rev. Mol. Cell Biol. 7, 347-358. doi: 10.1038/nrm1910

Evans, G. J., and Cousin, M. A. (2007). Activity-dependent control of slow synaptic vesicle endocytosis by cyclin-dependent kinase 5. J. Neurosci. 27, 401-411. doi: 10.1523/JNEUROSCI.3809-06.2007

Falace, A., Buhler, E., Fadda, M., Watrin, F., Lippiello, P., Pallesi-Pocachard, E., et al. (2014). TBC1D24, a protein involved in neurodevelopmental disorders, regulates cortical development through modulation of ARF6-dependent pathway. Proc. Natl. Acad. Sci. U S A 111, 2337-2342. doi: 10.1073/pnas. 1316294111
Falace, A., Filipello, F., La Padula, V., Vanni, N., Madia, F., De Pietri Tonelli, D. et al. (2010). TBC1D24, an ARF6-interacting protein, is mutated in familial infantile myoclonic epilepsy. Am. J. Hum. Genet. 87, 365-370. doi: 10.1016/j. ajhg.2010.07.020

Faúndez, V., Horng, J. T., and Kelly, R. B. (1997). ADP ribosylation factor 1 is required for synaptic vesicle budding in PC12 cells. J. Cell Biol. 138, 505-515. doi: $10.1083 /$ jcb.138.3.505

Fine, D., Flusser, H., Markus, B., Shorer, Z., Gradstein, L., Khateeb, S., et al. (2015). A syndrome of congenital microcephaly, intellectual disability and dysmorphism with a homozygous mutation in FRMD4A. Eur. J. Hum. Genet. 23, 1729-1734. doi: 10.1038/ejhg.2014.241

Fischer von Mollard, G., Stahl, B., Walch-Solimena, C., Takei, K., Daniels, L., Khoklatchev, A., et al. (1994). Localization of Rab5 to synaptic vesicles identifies endosomal intermediate in synaptic vesicle recycling pathway. Eur. J. Cell Biol. 65, 319-326.

Fletcher, A. I., Shuang, R., Giovannucci, D. R., Zhang, L., Bittner, M. A., and Stuenkel, E. L. (1999). Regulation of exocytosis by cyclin-dependent kinase 5 via phosphorylation of Munc18. J. Biol. Chem. 274, 4027-4035. doi: 10.1074/jbc. 274.7.4027

Geppert, M., Bolshakov, V. Y., Siegelbaum, S. A., Takei, K., De Camilli, P., Hammer, R. E., et al. (1994). The role of Rab3A in neurotransmitter release. Nature 369, 493-497. doi: 10.1038/369493a0

Geppert, M., Goda, Y., Stevens, C. F., and Südhof, T. C. (1997). The small GTPbinding protein Rab3A regulates a late step in synaptic vesicle fusion. Nature 387, 810-814. doi: 10.1038/42954

Giorgini, F., and Steinert, J. R. (2013). Rab11 as a modulator of synaptic transmission. Commun. Integr. Biol. 6:e26807. doi: 10.4161/cib.26807

Giovedi, S., Darchen, F., Valtorta, F., Greengard, P., and Benfenati, F. (2004a). Synapsin is a novel Rab3 effector protein on small synaptic vesicles. II. Functional effects of the Rab3A-synapsin I interaction. J. Biol. Chem. 279, 43769-43779. doi: 10.1074/jbc.m404168200

Giovedì, S., Vaccaro, P., Valtorta, F., Darchen, F., Greengard, P., Cesareni, G., et al. (2004b). Synapsin is a novel Rab3 effector protein on small synaptic vesicles. I. Identification and characterization of the synapsin I-Rab3 interactions in vitro and in intact nerve terminals. J. Biol. Chem. 279, 43760-43768. doi: 10.1074/jbc. $\mathrm{m} 403293200$

Homma, M., Nagashima, S., Fukuda, T., Yanagi, S., Miyakawa, H., Suzuki, E., et al. (2014). Downregulation of Centaurin gammalA increases synaptic transmission at Drosophila larval neuromuscular junctions. Eur. J. Neurosci. 40, 3158-3170. doi: 10.1111/ejn.12681

Hoopmann, P., Punge, A., Barysch, S. V., Westphal, V., Bückers, J., Opazo, F., et al. (2010). Endosomal sorting of readily releasable synaptic vesicles. Proc. Natl. Acad. Sci. U S A 107, 19055-19060. doi: 10.1073/pnas.1007037107

Kalscheuer, V. M., James, V. M., Himelright, M. L., Long, P., Oegema, R., Jensen, C., et al. (2016). Novel missense mutation A789V in IQSEC2 underlies X-linked intellectual disability in the MRX78 family. Front. Mol. Neurosci. 8:85. doi: 10 . 3389/fnmol.2015.00085

Kim, S., Ko, J., Shin, H., Lee, J. R., Lim, C., Han, J. H., et al. (2003). The GIT family of proteins forms multimers and associates with the presynaptic cytomatrix protein Piccolo. J. Biol. Chem. 278, 6291-6300. doi: 10.1074/jbc.m2122 87200

Kim, S. H., and Ryan, T. A. (2010). CDK5 serves as a major control point in neurotransmitter release. Neuron 67, 797-809. doi: 10.1016/j.neuron.2010. 08.003

Kim, S. H., and Ryan, T. A. (2013). Balance of calcineurin A $\alpha$ and CDK5 activities sets release probability at nerve terminals. J. Neurosci. 33, 8937-8950. doi: 10 . 1523/JNEUROSCI.4288-12.2013

Klassen, M. P., Wu, Y. E., Maeder, C. I., Nakae, I., Cueva, J. G., Lehrman, E. K., et al. (2010). An Arf-like small G protein, ARL-8, promotes the axonal transport of presynaptic cargoes by suppressing vesicle aggregation. Neuron 66, 710-723. doi: 10.1016/j.neuron.2010.04.033

Kononenko, N. L., and Haucke, V. (2015). Molecular mechanisms of presynaptic membrane retrieval and synaptic vesicle reformation. Neuron 85, 484-496. doi: 10.1016/j.neuron.2014.12.016

Krauss, M., Kinuta, M., Wenk, M. R., De Camilli, P., Takei, K., and Haucke, V. (2003). ARF6 stimulates clathrin/AP-2 recruiment to synaptic membranes by activating phosphatidylinositol 4-phosphate 5-kinase type gamma. J. Cell Biol. 162, 113-124. doi: $10.1083 /$ jcb.200301006 
Leitz, J., and Kavalali, E. T. (2015). $\mathrm{Ca}^{2+}$ dependence of synaptic vesicle endocytosis. Neuroscientist doi: 10.1177/1073858415588265 [Epub ahead of print].

Li, X., Sapp, E., Valencia, A., Kegel, K. B., Qin, Z. H., Alexander, J., et al. (2008). A function of huntingtin in guanine nucleotide exchange on Rab11. Neuroreport 19, 1643-1647. doi: 10.1097/WNR.0b013e328315cd4c

Liu, Y., Cheng, K., Gong, K., Fu, A. K., and Ip, N. Y. (2006). Pctaire1 phosphorylates $\mathrm{N}$-ethylmaleimide-sensitive fusion protein: implications in the regulation of its hexamerization and exocytosis. J. Biol. Chem. 281, 9852-9858. doi: 10.1074/jbc.m513496200

Marks, B., and McMahon, H. T. (1998). Calcium triggers calcineurin-dependent synaptic vesicle recycling in mammalian nerve terminals. Curr. Biol. 18, 740-749. doi: 10.1016/s0960-9822(98)70297-0

Marra, V., Burden, J. J., Thorpe, J. R., Smith, I. T., Smith, S. L., Häusser, M., et al. (2012). A preferentially segregated recycling vesicle pool of limited size supports neurotransmission in native central synapses. Neuron 76, 579-589. doi: 10.1016/j.neuron.2012.08.042

McLinden, K. A., Trunova, S., and Giniger, E. (2012). At the fulcrum in health and disease: $\mathrm{Cdk} 5$ and the balancing acts of neuronal structure and physiology. Brain Disord. Ther. 1:001. doi: 10.4172/2168-975x.s1-001

Mitra, A., Mitra, S. S., and Tsien, R. W. (2011). Heterogeneous reallocation of presynaptic efficacy in recurrent excitatory circuits adapting to inactivity. Nat. Neurosci. 15, 250-257. doi: 10.1038/nn.3004

Miyamoto, Y., Yamamori, N., Torii, T., Tanoue, A., and Yamauchi, J. (2014). Rab35, acting through ACAP2 switching off Arf6, negatively regulates oligodendrocyte differentiation and myelination. Mol. Biol. Cell. 25, 1532-1542. doi: 10.1091/mbc.e13-10-0600

Montesinos, M. S., Dong, W., Goff, K., Das, B., Guerrero-Given, D., Schmalzigaug, R., et al. (2015). Presynaptic deletion of GIT proteins results in increased synaptic strength at a mammalian central synapse. Neuron 88, 918-925. doi: 10. 1016/j.neuron.2015.10.042

Pavlos, N. J., Grønborg, M., Riedel, D., Chua, J. J., Boyken, J., Kloepper, T. H., et al. (2010). Quantitative analysis of synaptic vesicle Rabs uncovers distinct yet overlapping roles for Rab3a and Rab27b in $\mathrm{Ca}^{2+}$-triggered exocytosis. J. Neurosci. 30, 13441-13453. doi: 10.1523/JNEUROSCI.0907-10.2010

Pavlos, N. J., and Jahn, R. (2011). Distinct yet overlapping roles of Rab GTPases on synaptic vesicles. Small GTPases 2, 77-81. doi: 10.4161/sgtp.2.2. 15201

Peng, Y. R., Hou, Z. H., and Yu, X. (2013). The kinase activity of EphA4 mediates homeostatic scaling-down of synaptic strength via activation of Cdk5. Neuropharmacology 65, 232-243. doi: 10.1016/j.neuropharm.2012. 10.012

Podufall, J., Tian, R., Knoche, E., Puchkov, D., Walter, A. M., Rosa, D., et al. (2014). A presynaptic role for the cytomatrix protein GIT in synaptic vesicle recycling. Cell Rep. 7, 1417-1425. doi: 10.1016/j.celrep.2014.04.051

Rauch, A., Wieczorek, D., Graf, E., Wieland, T., Endele, S., Schwarzmayr, T., et al. (2012). Range of genetic mutations associated with severe nonsyndromic sporadic intellectual disability: an exome sequencing study. Lancet 380, 1674-1682. doi: 10.1016/S0140-6736(12)61480-9

Rizzoli, S. O. (2014). Synaptic vesicle recycling: steps and principles. EMBO J. 33, 788-822. doi: 10.1002/embj.201386357

Sakane, A., Manabe, S., Ishizaki, H., Tanaka-Okamoto, M., Kiyokage, E., Toida, K., et al. (2006). Rab3 GTPase-activating protein regulates synaptic transmission and plasticity through the inactivation of Rab3. Proc. Natl. Acad. Sci. US A 103, 10029-10034. doi: 10.1073/pnas.0600304103

Schlüter, O. M., Schmitz, F., Jahn, R., Rosenmund, C., and Südhof, T. C. (2004). A complete genetic analysis of neuronal Rab3 function. J. Neurosci. 24, 6629-6637. doi: 10.1523/JNEUROSCI.1610-04.2004

Seeburg, D. P., Feliu-Mojer, M., Gaiottino, J., Pak, D. T., and Sheng, M. (2008). Critical role of CDK5 and Polo-like kinase 2 in homeostatic synaptic plasticity during elevated activity. Neuron 58, 571-583. doi: 10.1016/j.neuron.2008. 03.021

Seixas, E., Barros, M., Seabra, M. C., and Barral, D. C. (2013). Rab and Arf proteins in genetic diseases. Traffic 14, 871-885. doi: 10.1111/tra.12072

Shah, K., and Lahiri, D. K. (2014). Cdk5 activity in the brain - multiple paths of regulation. J. Cell Sci. 127, 2391-2400. doi: 10.1242/jcs.147553

Sheng, Y., Zhang, L., Su, S. C., Tsai, L. H., and Julius Zhu, J. (2015). Cdk5 is a new rapid synaptic homeostasis regulator capable of initiating the early alzheimer-like pathology. Cereb. Cortex doi: 10.1093/cercor/bhv032 [Epub ahead of print].

Shoubridge, C., Tarpey, P. S., Abidi, F., Ramsden, S. L., Rujirabanjerd, S., Murphy, J. A., et al. (2010). Mutations in the guanine nucleotide exchange factor gene IQSEC2 cause nonsyndromic intellectual disability. Nat. Genet. 42, 486-488. doi: $10.1038 /$ ng. 588

Shuang, R., Zhang, L., Fletcher, A., Groblewski, G. E., Pevsner, J., and Stuenkel, E. L. (1998). Regulation of Munc-18/syntaxin 1A interaction by cyclindependent kinase 5 in nerve endings. J. Biol. Chem. 273, 4957-4966. doi: 10. 1074/jbc.273.9.4957

Shimizu, H., Kawamura, S., and Ozaki, K. (2003). An essential role of Rab5 in uniformity of synaptic vesicle size. J. Cell Sci. 116, 3583-3590. doi: 10.1242/jcs. 00676

Star, E. N., Newton, A. J., and Murthy, V. N. (2005). Real-time imaging of Rab3a and Rab5a reveals differential roles in presynaptic function. J. Physiol. 569, 103-117. doi: 10.1113/jphysiol.2005.092528

Steinert, J. R., Campesan, S., Richards, P., Kyriacou, C. P., Forsythe, I. D., and Giorgini, F. (2012). Rab11 rescues synaptic dysfunction and behavioural deficits in a Drosophila model of Huntington's disease. Hum. Mol. Genet. 21, 2912-2922. doi: 10.1093/hmg/dds117

Stenmark, H. (2009). Rab GTPases as coordinators of vesicle traffic. Nat. Rev. Mol. Cell Biol. 10, 513-525. doi: 10.1038/nrm2728

Su, S. C., Seo, J., Pan, J. Q., Samuels, B. A., Rudenko, A., Ericsson, M., et al. (2012). Regulation of $\mathrm{N}$-type voltage-gated calcium channels and presynaptic function by cyclin-dependent kinase 5. Neuron 75, 675-687. doi: 10.1016/j.neuron.2012. 06.023

Su, S. C., and Tsai, L. H. (2011). Cyclin-dependent kinases in brain development and disease. Annu. Rev. Cell Dev. Biol. 27, 465-491. doi: 10.1146/annurevcellbio-092910-154023

Sudhof, T. C. (2004). The synaptic vesicle cycle. Annu. Rev. Neurosci. 27, 509-547. doi: 10.1146/annurev.neuro.26.041002.131412

Tagliatti, E., Fadda, M., Falace, A., Benfenati, F., and Fassio, A. (2016). Arf6 regulates the cycling and the readily releasable pool of synaptic vesicles at hippocampal synapse. Elife 5:e10116. doi: 10.7554/elife.10116

Takamori, S., Holt, M., Stenius, K., Lemke, E. A., Grønborg, M., Riedel, D., et al. (2006). Molecular anatomy of a trafficking organelle. Cell 127, 831-846. doi: 10. 1016/j.cell.2006.10.030

Taniguchi, M., Taoka, M., Itakura, M., Asada, A., Saito, T., Kinoshita, M., et al. (2007). Phosphorylation of adult type Sept5 (CDCrel-1) by cyclin-dependent kinase 5 inhibits interaction with syntaxin-1. J. Biol. Chem. 282, 7869-7876. doi: 10.1074/jbc.m609457200

Uytterhoeven, V., Kuenen, S., Kasprowicz, J., Miskiewicz, K., and Verstreken, P. (2011). Loss of Skywalker reveals synaptic endosomes as sorting stations for synaptic vesicle proteins. Cell 145, 117-132. doi: 10.1016/j.cell.2011. 02.039

Valtorta, F., and Benfenati, F. (1994). Protein phosphorylation and the control of exocytosis in neurons. Ann. N Y Acad. Sci. 710, 347-355. doi: 10.1111/j.17496632.1994.tb26641.x

Verstegen, A. M., Tagliatti, E., Lignani, G., Marte, A., Stolero, T., Atias, M., et al. (2014). Phosphorylation of synapsin I by cyclin-dependent kinase-5 sets the ratio between the resting and recycling pools of synaptic vesicles at hippocampal synapses. J. Neurosci. 34, 7266-7280. doi: 10.1523/JNEUROSCI. 3973-13.2014

Wilhelm, B. G., Mandad, S., Truckenbrodt, S., Kröhnert, K., Schäfer, C., Rammner, B., et al. (2014). Composition of isolated synaptic boutons reveals the amounts of vesicle trafficking proteins. Science 344, 1023-1028. doi: 10. $1126 /$ science. 1252884

Wu, L. G., Hamid, E., Shin, W., and Chiang, H. C. (2014). Exocytosis and endocytosis: modes, functions and coupling mechanisms. Annu. Rev. Physiol. 76, 301-331. doi: 10.1146/annurev-physiol-021113-170305

Wu, Y. E., Huo, L., Maeder, C. I., Feng, W., and Shen, K. (2013). The balance between capture and dissociation of presynaptic proteins controls the spatial distribution of synapses. Neuron 78, 994-1011. doi: 10.1016/j.neuron.2013. 04.035

Wucherpfennig, T., Wilsch-Bräuninger, M., and González-Gaitán, M. (2003). Role of Drosophila Rab5 during endosomal trafficking at the synapse and evoked neurotransmitter release. J. Cell Biol. 161, 609-624. doi: 10.1083/jcb.2002 11087 
Xiong, Y., Yuan, C., Chen, R., Dawson, T. M., and Dawson, V. L. (2012). ArfGAP1 is a GTPase activating prote in for LRRK2: reciprocal regulation of ArfGAP1 by LRRK2. J. Neurosci. 32, 3877-3886. doi: 10.1523/JNEUROSCI.4566-11.2012

Yamaguchi, K., Tanaka, M., Mizoguchi, A., Hirata, Y., Ishizaki, H., Kaneko, K., et al. (2002). A GDP/GTP exchange protein for the Rab3 small G protein family up-regulates a postdocking step of synaptic exocytosis in central synapses. Proc. Natl. Acad. Sci. U S A 99, 14536-14541. doi: 10.1073/pnas.212511399

Yun, H. J., Kim, H., Ga, I., Oh, H., Ho, D. H., Kim, J., et al. (2015). An early endosome regulator, Rab5b, is an LRRK2 kinase substrate. J. Biochem. 157, 485-495. doi: 10.1093/jb/mvv005
Conflict of Interest Statement: The authors declare that the research was conducted in the absence of any commercial or financial relationships that could be construed as a potential conflict of interest.

Copyright (c) 2016 Fassio, Fadda and Benfenati. This is an open-access article distributed under the terms of the Creative Commons Attribution License (CC BY). The use, distribution and reproduction in other forums is permitted, provided the original author(s) or licensor are credited and that the original publication in this journal is cited, in accordance with accepted academic practice. No use, distribution or reproduction is permitted which does not comply with these terms. 